\title{
An Account for Dark Matter and Dark Energy by a Combined Spacetime Four-Manifold
}

\author{
Gregory L. Light \\ Department of Management, Providence College \\ 1 Cunningham Square, Providence, Rhode Island 02918, USA \\ Tel: 1-401-865-2662Ｅ-mail: glight@providence.edu
}

Received: July 18, 2011 Accepted: August 2, $2011 \quad$ Published: November 1, 2011

doi:10.5539/apr.v3n2p130

URL: http://dx.doi.org/10.5539/apr.v3n2p130

\begin{abstract}
We explain dark matter and dark energy by our previous construct of a combined spacetime 4-manifold "U3," which is the graph of a diffeomorphism from the particle universe "U1" to the wave universe "U2." Dark matter and dark energy exist respectively as standing waves and traveling waves in U2, while ordinary matter and light are "particle waves" in U3. As such, our model is founded upon the wave-particle duality.
\end{abstract}

Keywords: Quantum tunneling entanglement computing, Vacuum energy, Cosmological constant, P-branes, AdS/CFT, Kähler manifolds, Particle-wave duality, Bimetric gravitation, Diagonal map

\section{Introduction}

This paper is based on our previous construct of a "combined spacetime 4-manifold" (see Light, 2011) to explain the observed phenomena of dark matter and dark energy (see, e.g., [Amendola, L., Tsujikawa, S., 2010] for a summary of the existing theories and observations). Our model originated from the idea of a diagonal map in differential geometry. We relate a particle universe $\mathrm{M}^{[1]}$ to a wave universe $\mathrm{M}^{[2]}$ and combine them into one common universe $\mathrm{M}^{[3]}$.

Over the years gravitational anomalies have brought up a number of theories or themes of study, such as dual mass (cf. e.g., [Cates, et al., 1988]), shadow matter (cf. e.g., [Tosa, Okubo, 1987]), gravitational dyon (cf. e.g., [Tübingen, 1987]), fifth force (cf. e.g., [Riveros, et al., 1989]), dark matter (cf. e.g., [Anderson, et al., 1989]), and dark energy. That dark matter and dark energy have entered the center stage of physics is attributed to the existence of indisputable cosmological observations. Unexplained gravitational accelerations of astral bodies cemented the idea of dark matter, and accelerated expansion of the Universe indicated the existence of dark energy.

Before proceeding to our analysis in Section 2, we present a general description of our model and a brief account of its relations to existing theories. We hypothesize that our recognized Universe is made up of two coincidental copies of spacetimes, as maybe pictured as two identical stamps glued together. The visible copy contains particles engaging in all the familiar four forces, and the invisible copy contains electromagnetic waves engaging only in gravity. We united the two sets of gravitational motions into one by an observation of the form invariance of the time $\times$ time components of the metric tensors as evolved from two independent sets of Einstein Field Equations, each with its own distinct gravitational constant. After deriving the value of the gravitational constant of the wave universe in particular, we found it to be so large as to cause easily an astronomically-sized black hole. We thus contemplate the possibility that a formation of such a "Black Hole" (capitalized for subsequent referencing) gave the Big Bang that created our visible universe of particles. To be specific, the infinite spacetime curvature at the center of the Black Hole altered the hitherto pure electromagnetic waves into photon-waves and the set of photon-waves developed and inflated into a distinct universe of particle-waves (á la [Feynman, 1963]). By an observation of Feynman's analysis of the electromagnetic mass of an electron (ibid.), we set the energy distribution of a (particle, wave) to be $(0.75,0.25)$. We propose that the dark matter and dark energy are respectively the standing and traveling waves in the Black Hole that did not turn into photon-waves following the Big Bang. Fanciful as the above description might sound, our model of a combined spacetime 4-manifold of the Universe actually has already been well treated by standard physics, as explained below. 
First, the basic quantum mechanical setup of brakets in a Hilbert space effectively provided a vehicle to analyze our particle-waves, except for the difference that all energies were quantized as particles and waves were relegated to probabilities. Schrödinger's wave equation with the imaginary number $i$ formally began to analyze the wave dynamics in our Black Hole, which, by the way, had actually been done by Maxwell isomorphically in real terms more than sixty years ago. Dirac gave a description of our electron-wave and showed how a particle-wave rotated within our combined 4-manifold. His well-known interpretations of the positron and vacuum differed from ours, however. We consider a positron as a particle-wave resulting from an interchange of the ordering in the cross product of the electric and magnetic fields in the Black Hole and vacuum as a region occupied exclusively by the dark energy as cast in our model. The Standard Model provided critical treatments of our wave universe in conjunction with our particle universe, establishing fundamental laws of particle formations and interactions. Concerning gravity, the Calabi-Yau manifolds with three spatial complex dimensions, $Y \equiv\left\{\left(\widetilde{x}_{1}, i \widetilde{x}_{2}\right)\right\} \times\left\{\left(\widetilde{y}_{1}, \tilde{y}_{2}\right)\right\} \times\left\{\left(\widetilde{z}_{1}, i \widetilde{z}_{2}\right)\right\}$, can specialize into $Y \equiv\left\{\left(0, i \widetilde{x}_{2}\right)\right\} \times\left\{\left(0, i \widetilde{y}_{2}\right)\right\} \times\left\{\left(0, \widetilde{z}_{2}\right)\right\}$ for our Black Hole. In addition, if we identify our electromagnetic waves with some particular 3-branes and embed our combined 4-manifold, $\{(t, x, y, z)\} \times\{(i t, i x, i y, i z)\}$, into $\{(t, x, y, z)\} \times\{i t\} \times Y$, then our model becomes a special case of the 11-dimensional M-theory by Witten. In passing, we note that dark matter in string theories has been considered as supersymmetric particles, and dark energy as that resulting from manifold compactification.

The following Section 2 will first introduce our construct of a combined 4-manifold as based on a pair of Einstein Field Equations. Then we unify the two sets of gravitational motions by establishing three gravitational constants, $G^{[1]}, G^{[2]}$, and $G^{[3]}$, respectively of $\mathrm{M}^{[1]}, \mathrm{M}^{[2]}$, and $\mathrm{M}^{[3]}$. With $G^{[2]}$ ascertained, we proceed to examine the possible existence of dark matter and dark energy in $\mathrm{M}^{[2]}$. We distinguish our approach by a 4-dimensional Riemannian geometry that adheres to Einstein's General Relativity and is free from proposing new particles. Finally Section 3 will conclude with a few summary remarks.

\section{Analysis}

We begin with a definition of a combined spacetime 4-manifold

$$
\mathbf{M}^{[3]}:=\left\{\left(p^{[1]}, p^{[2]}\right) \in \mathbf{M}^{[1]} \times \mathbf{M}^{[2]} \mid h\left(p^{[1]}\right)=p^{[2]}, h=\text { any diffeomorp hism }\right\},
$$

where $\mathrm{M}^{[1]}$ contains particles and $\mathrm{M}^{[2]}$, electromagnetic waves as derived from Maxwell equations for free spaces. We note that there can exist two kinds of waves in $\mathrm{M}^{[1]}$ : traveling and standing. Consider the superposition of two traveling waves at speed $\mathrm{c} \equiv \omega / \mathrm{k}$ in opposite directions:

$$
\cos (k x-\omega t)+\cos (-k x-\omega t)=2 \cos k x \cos k c t,
$$

i.e., resulting in a standing wave.

By General Relativity, $\mathrm{M}^{[1]}$ and $\mathrm{M}^{[2]}$ observe Einstein Field Equations,

$$
R_{\mu \nu}^{[i]}-\frac{1}{2} R^{[i]} g_{\mu \nu}^{[i]}=-\frac{8 \pi G^{[i]}}{c^{2}} T_{\mu \nu}^{[i]}, \quad i=1,2, \text { and }
$$

$G^{[i]}=$ the gravitational constant of $\mathrm{M}^{[\mathrm{i}]}$.

But

$$
g^{[i]}{ }_{11}=1-\frac{2 G^{[i]} M^{[i]}}{r c^{2}} \forall i=1,2,3,
$$

and $g^{[3]}$ being the inner product of the direct sum of the tangent spaces

$$
T_{p^{[1]}} \mathbf{M}^{[1]} \oplus T_{p^{[2]}} \mathbf{M}^{[2]}
$$

suggests from standard linear algebra that 


$$
g^{[3]}=a \cdot g^{[1]}+b \cdot g^{[2]}
$$

for some constants $\mathrm{a}, \mathrm{b}>0$. Substituting Equation (2.3) into (2.5), we have

$$
\begin{aligned}
& a=\frac{G^{[2]}}{G^{[1]}+G^{[2]}}, \\
& b=\frac{G^{[1]}}{G^{[1]}+G^{[2]}}, \quad \text { and } \\
& G^{[3]}=\frac{G^{[1]} G^{[2]}}{G^{[1]}+G^{[2]}} .
\end{aligned}
$$

Passing to the Newtonian limit, we then have

$$
m^{[3]} \mathbf{a}^{[3]}=-\left[\left(\frac{G^{[2]}}{G^{[1]}+G^{[2]}}\right)\left(\frac{G^{[1]} M^{[1]} m^{[1]}}{\|\mathbf{r}\|^{2}}\right)+\left(\frac{G^{[1]}}{G^{[1]}+G^{[2]}}\right)\left(\frac{G^{[2]} M^{[2]} m^{[2]}}{\|\mathbf{r}\|^{2}}\right)\right] \cdot \frac{\mathbf{r}}{\|\mathbf{r}\|},
$$

or

$$
\mathbf{a}^{[3]}=-\frac{G^{[3]} M^{[3]}}{\|\mathbf{r}\|^{2}}\left(\frac{M^{[1]}}{M^{[3]}} \cdot \frac{m^{[1]}}{m^{[3]}}+\frac{M^{[2]}}{M^{[3]}} \cdot \frac{m^{[2]}}{m^{[3]}}\right) \frac{\mathbf{r}}{\|\mathbf{r}\|},
$$

Where $m^{[3]}=m^{[1]}+m^{[2]}$ and $M^{[3]}=M^{[1]}+M^{[2]}$. That is, we have unified the two sets of gravitational motions.

Setting

$$
\frac{M^{[1]}}{M^{[3]}}=\frac{m^{[1]}}{m^{[3]}} \equiv \mu_{1} \in(0,1)
$$

we can rewrite the above Equation (2.8) as

$$
\mathbf{a}^{[3]}=-\frac{G^{[3]}\left[M^{[3]}\left(\boldsymbol{\mu}_{1}^{2}+\left(1-\boldsymbol{\mu}_{1}\right)^{2}\right)\right]}{\|\mathbf{r}\|^{2}} \frac{\mathbf{r}}{\|\mathbf{r}\|} \equiv-\frac{G^{[3]} \hat{M}}{\|\mathbf{r}\|^{2}} \frac{\mathbf{r}}{\|\mathbf{r}\|},
$$

where $G^{[3]}=$ the recognized gravitational constant, and $\hat{M}=$ the laboratory-measured mass. That is,

$$
\begin{gathered}
M^{[3]}=\frac{\hat{M}}{\mu_{1}^{2}+\left(1-\mu_{1}\right)^{2}}, \\
M^{[1]}=\frac{\hat{M} \mu_{1}}{\mu_{1}^{2}+\left(1-\mu_{1}\right)^{2}} \equiv \hat{M} \phi^{[1]}, \text { and } \\
M^{[2]}=\frac{\hat{M}\left(1-\mu_{1}\right)}{\mu_{1}^{2}+\left(1-\mu_{1}\right)^{2}} \equiv \hat{M} \phi^{[2]} .
\end{gathered}
$$

Concerning $\mu_{1} \equiv \frac{M^{[1]}}{M^{[3]}}$, we consider the discrepancy in the electromagnetic mass of an electron as measured in 
a stationary state versus in a moving state with a constant velocity of $\|\mathbf{V}\|<c$. In [Feynman, II-28-4, 1963], one finds (cf. also [Moylan, 1995] for this well-known problem)

$$
m_{V=0}=\frac{3}{4} m_{V \neq 0} .
$$

Since electromagnetic forces take place by exchanging virtue particles in $\mathrm{M}^{[1]}$ and yet motions necessarily take place in $\mathrm{M}^{[3]}$, we attribute $m_{V=0}$ to $\mathrm{M}^{[1]}$ and $m_{V \neq 0}$ to $\mathrm{M}^{[3]}$, so that

$$
\mu_{1}=\frac{3}{4}
$$

As such, we have

$$
\phi^{[1]}=1.2 \text { and } \phi^{[2]}=0.4
$$

We next derive the value of $G^{[2]}$, but first we define the following notations:

$$
\ddot{h} \equiv \frac{\text { Planck constant } h}{\text { second }^{2}} ; N L T \equiv \text { nonlinear terms related } \text {. }
$$

In order to apply General Relativity in our derivation, we set the Planck length $\lambda_{P}: \approx 10^{-35}$ meter as the lower limit of electromagnetic wave lengths under consideration, i.e., $\lambda \in\left[\lambda_{P}, \infty\right)$, or equivalently, $\boldsymbol{v} \equiv \frac{c}{\lambda} \in\left(0 \mathrm{~Hz}, 10^{43} \mathrm{~Hz} \equiv \boldsymbol{v}_{\text {Planck }}\right)$. Thus, let $E_{j}^{\left[{ }^{[1]}\right.}$ be the energy of a photon $\mathrm{j}$ with frequency $\boldsymbol{v}_{j}^{[1]} \in\left(0 H z, \boldsymbol{v}_{\text {Planck }}\right)$ as observed from a laboratory frame $S^{[1]}$ in $\mathrm{M}^{[1]}$. Consider $E_{j}^{[2]}\left(\equiv \hat{E}_{j} \boldsymbol{\phi}^{[2]}\right.$, recall the definition in (2.13), where $\hat{E}_{j}$ is the laboratory-measured energy of $\mathrm{j}$ ) within its wave length $\lambda_{j}$, i.e., $E_{j}^{[2]}$ as contained in a ball $B$ of radius $\frac{\lambda_{j}}{2}$, and consider a reference frame $S^{[2]}$ on the boundary of $B$. Since the gravitational effect of $E_{j}^{[2]}$ on $S^{[2]}$ is as if the ball $B$ of energy $E_{j}^{[2]}$ were concentrated at the ball center, we have

$$
g_{11}{ }^{[2]}=1-\frac{2 G^{[2]} E_{j}^{[2]}}{\frac{\lambda_{j}}{2} \cdot c^{4}} \equiv 1-\frac{4 G^{[2]} E_{j}^{[2]} \boldsymbol{v}_{j}^{[1]}}{c^{5}} \text {. }
$$

Since the frequency $\boldsymbol{v}_{j}{ }^{[2]}$ of $E_{j}^{[2]}$ relative to frame $S^{[2]}$ is exactly 1 cycle, we have

$$
\boldsymbol{v}_{j}^{[2]}=\frac{1(\text { cycle })}{\text { second }},
$$

so that 


$$
\begin{aligned}
& g_{11}{ }^{[2]}:=\left(\frac{\partial t_{0}{ }^{[2]}}{\partial t_{0}{ }^{[1]}}\right)^{2}:=\lim _{\Delta t_{0}{ }^{1]} \rightarrow 0}\left(\frac{\Delta t_{0}{ }^{[2]}}{\Delta t_{0}{ }^{[1]}}\right)^{2}=\left(\frac{\Delta t_{0}{ }^{[2]}}{\Delta t_{0}{ }^{[1]}=1 \text { second }}\right)^{2}-N L T \equiv\left(\frac{\boldsymbol{v}_{j}^{[1]}}{\boldsymbol{v}_{j}{ }^{[2]}}\right)^{2}-N L T \\
& \equiv\left(\frac{\boldsymbol{v}_{j}{ }^{[1]}}{1 / \text { second }}\right)^{2}-N L T=\boldsymbol{v}_{j}{ }^{[1]^{2}} \operatorname{second}^{2}-N L T=1-\frac{4 G^{[2]} E_{j}{ }^{[2]} \boldsymbol{v}_{j}{ }^{[1]}}{c^{5}} \quad(\text { from }(2.18)) .
\end{aligned}
$$

Thus,

$$
\boldsymbol{v}_{j}{ }^{[1]} \operatorname{second}^{2}-\frac{N L T+1}{\boldsymbol{v}_{j}{ }^{[1]}}=-\frac{4 G^{[2]} E^{[2]}}{c^{5}},
$$

where NLT involving the Planck length is outside the above indicated domain of calculus. Letting $\boldsymbol{v}_{j}{ }^{[1]}$ approach $\boldsymbol{v}_{\text {Planck }}$ in Equation (2.21), we see that the positive left-hand-side cannot equal the negative right-hand-side. We now need examine closely the isomorphism:

$$
\cos (k x-\omega t) \cong e^{-i(\omega t-k x)}
$$

This is a covering map of $\mathrm{R}$ onto the circle $\mathbf{S}^{\mathbf{1}}$. Setting $\omega=2 \pi$ radians/second for convenience, we see that as $\mathrm{t}$ increases from 0 second to 1 second linearly along $\mathrm{R},(i \cdot t)$ increases from $0 \cdot(i$ second $)$ to $1 \cdot(i$ second $)$ clockwise along $\mathbf{S}^{\mathbf{1}}$ so that $-\boldsymbol{\omega} \cdot i t=-(2 \pi$ radians $/$ second $) \cdot(i$ second $)=-2 \pi i$ and $e^{-2 \pi i}=1$ cycle clockwise. I.e., while $\mathrm{t}$ registers time along $\mathrm{R},(i \cdot t)$ registers time along $\mathrm{S}^{1}$. Simply said, if $\mathrm{t}=2011$ years, then $i t=2011 i$ years would mean that other than the 12-month progression there had been no changes year after year since $\mathrm{t}=0$ year. Since electromagnetic waves by nature are cyclic, the proper unit of time must be ( $i$ second). Thus, we revise Equation (2.19) to

$$
\boldsymbol{v}_{j}{ }^{[2]}=\frac{1(\text { cycle })}{i \cdot \text { second }} .
$$

Consequently, Equation (2.21) becomes

$$
-\boldsymbol{v}_{j}{ }^{[1]} \text { second }^{2}-\frac{N L T+1}{\boldsymbol{v}_{j}{ }^{[1]}}=-\frac{4 G^{[2]} E^{[2]}}{c^{5}},
$$

or

$$
\frac{c^{5}}{4 G^{[2]}}\left(\boldsymbol{v}_{j}^{[1]} \operatorname{second}^{2}+\frac{N L T+1}{\boldsymbol{v}_{j}^{[1]}}\right)=E_{j}^{[2]} \equiv \hat{E}_{j} \boldsymbol{\phi}^{[2]}
$$

or

$$
\begin{aligned}
& \hat{E}_{j}=\left(\frac{c^{5} \text { second }^{2}}{4 \mathrm{G}^{[2]} \boldsymbol{\phi}^{[2]}}\right) \cdot \boldsymbol{v}_{j}^{[1]}+\left(\frac{c^{5}}{4 \mathrm{G}^{[2]} \boldsymbol{\phi}^{[2]}}\right) \cdot \frac{N L T+1}{\boldsymbol{v}_{j}^{[1]}} \\
& \equiv h \cdot \boldsymbol{v}_{j}^{[1]}+\ddot{h} \cdot \frac{N L T+1}{\boldsymbol{v}_{j}^{[1]}}
\end{aligned}
$$

where 


$$
\ddot{h} \cdot \frac{N L T+1}{\boldsymbol{v}_{j}^{[1]}} \equiv \Delta \hat{E}_{j}
$$

is the uncertainty energy due to the fact that $g_{11}{ }^{[2]}=1-\frac{4 G^{[2]} E_{j}{ }^{[2]} \boldsymbol{v}_{j}{ }^{[1]}}{c^{5}}$, being a derivative, contains quantum uncertainties as $\Delta t_{0}^{[1]} \rightarrow 0$. Thus, from Equations (2.25), we have

$$
G^{[2]}=\frac{c^{5}}{4 \ddot{h}_{\phi}^{[2]}}
$$

Recalling from Equation (2.16) that $\phi^{[2]}=0.4$, we then have

$$
G^{[2]}=\frac{c^{5}}{1.6 \ddot{h}} \approx 2.3 \times 10^{75} \times \frac{\text { meter }^{3}}{\text { kilogram } \cdot \text { second }^{2}} \approx 10^{85} G^{[1]} \approx 10^{85} G^{[3]} .
$$

We now calculate the Schwarzschild radius for $\mathrm{M}^{[2]}$ by solving

$$
g_{11}^{[2]}=0=1-\frac{2 G^{[2]} M^{[2]}}{R^{[2]} c^{2}}
$$

for $R^{[2]}$. From Equations (2.13) and (2.16), we have

$$
\frac{M^{[2]}}{M^{[1]}}=\frac{\phi^{[2]}}{\phi^{1}}=\frac{0.4}{1.2}=\frac{1}{3} .
$$

Since $M^{[1]} \equiv \hat{M} \phi^{[1]}=$ the observed mass of the Universe $\approx 10^{51} \mathrm{~kg} \times 1.2$, we have

$$
g_{11}^{[2]}=0=1-\frac{2 \times 6.7 \times 10^{-11} \times 10^{85} \times 1.2 \times 10^{51} \times(1 / 3)}{R^{[2]} \times 9 \times 10^{16}} .
$$

Thus,

$$
R^{[2]} \approx 10^{108} \text { meters, }
$$

which is greater than the recognized radius for $\mathrm{M}^{[1]}, 10^{26}$ meters. As such, we consider the possibility that prior to the Big Bang there had only been $\mathrm{M}^{[2]}$; because of the large $G^{[2]}$ an astronomical black hole $B \subset M^{[2]}$ came into being - - the Big Bang. B transformed some of the therein contained electromagnetic waves into photon-waves, and the collection of photons formed $\mathrm{M}^{[1]}$. (cf. [Frolov et al. 1990] for the possibility that a black hole can give rise to a macroscopic universe), where all particles ultimately were made up of photons via $\gamma+\gamma \rightarrow$ electron $e^{-}+$positron $e^{+}$, or superposition of electromagnetic waves, manifested as matter waves by de Broglie.

In conclusion, there exist four kinds of objects in the Universe $\mathrm{M}^{[3]}$ : photons and matter in $\mathrm{M}^{[1]}$ (both of which carry $(1 / 3)$ of their energies in $\mathrm{M}^{[2]}$ ), and the non-transformed electromagnetic waves in $B \subset M^{[2]}$, which can be traveling or standing and are respectively shown as dark energy or dark matter in $\mathrm{M}^{[1]}$.

\section{Summary remarks}

We complete this paper with a summary account of some of the distinct features of our model of a combined spacetime 4-manifold of the Universe. (1) Foremost, our model is constructed upon two sets of Einstein Field Equations, involving three gravitational constants and having no cosmological constants. (2) Our model makes quantum black holes in the particle universe gateways to and from the Black Holes in the wave universe, so that 
any point particle-wave such as the electron-wave does not have an infinite energy density. Point particle-waves by their own gravity cause quantum black holes, which continually send them "back" to the Black Hole in the wave universe to "resume" as pure invisible electromagnetic waves, although only to re-emerge in the particle universe according to the quantum creation and annihilation operators. (3) Our combined 4-manifold corresponds directly to the particle-wave duality. Our model provides energy contents to the probabilistic wavefunctions. The quotient spacetimes in $B \subset M^{[2]}$ explain why the complex number $i$ is indispensable in quantum mechanics (cf. [Barbour, 1993]) and why quantum entanglements are possible. In [Light, 2011] we used the quotient-spacetime geometry to explain the double-slit experiment. We showed that the ejected photon-wave discerned the setup of the slits by the distance in the Black Hole of the wave universe, not by the distance in the particle universe. This long-distance information sharing in the particle universe via the equivalence classes of the spacetimes in the Black Hole of the wave universe should play an important role in quantum computing. To go one step further, we suggest experiments on long-distance quantum tunneling as a critical test of our model.

\section{References}

Amendola, L. \& Tsujikawa, S. (2010). Dark energy, theory and observations. Cambridge: Cambridge University Press.

Anderson, J. D. et al. (1989). Bounds on dark matter in solar orbit. The Astrophysical Journal, 342, 539-544. http://dx.doi.org/10.1086/167614

Barbour, J. B. (1993). Time and complex numbers in canonical quantum gravity. Physical Review D, 47, 5422 $(1-27)$.

Cates, W. et al. (1988). Existence of dual mass: experimental evidence. General Relativity and Gravitation, 20, 1055-1066. http://dx.doi.org/10.1007/BF00759026

Feynman, R. P. et al. (1963). The Feynman lectures on physics. Reading: Addison-Wesley.

Frolov, V. P. et al. (1990). Black holes as possible sources of closed and semiclosed worlds. Physical Review D, 41, $383(1-11)$.

Light, G. L. (2011). General relativity extended. In A. A. Kishk (Ed.). Electromagnetic waves propagation in complex matter (pp. 157-184). Rijeka: InTech, Open Access Publisher.

Moylan, P. (1995). An elementary account of the factor of $4 / 3$ in the electromagnetic mass. American Journal of Physics, 63, 818-820. http://dx.doi.org/10.1119/1.17806

Riveros, C. et al. (1989). On differential fifth force measurements. Physics Letters A, 136, 343-347. http://dx.doi.org/10.1016/0375-9601(89)90412-X

Tosa, Y. \& Okubo, S. (1987). Systematic search for anomaly-free theories. Physical Review D, 36, 2484-2505. http://dx.doi.org/10.1103/PhysRevD.36.2484

Tübingen, W. C. (1987). The gravitational field of an abelian dyon in the bi-metric theory of gravitation. Acta Physica Slovaca, 37, 369-375. 